\title{
MCAO for Gemini South
}

\section{Brent L. Ellerbroek, Francois J. Rigaut, Brian J. Bauman, Corinne Boyer, Stephen L. Browne, et al.}

Brent L. Ellerbroek, Francois J. Rigaut, Brian J. Bauman, Corinne Boyer, Stephen L. Browne, Richard A. Buchroeder, James W. Catone, Paul Clark, Celine d'Orgeville, Donald T. Gavel, Glen Herriot, Mark R. Hunten, Eric James, Edward J. Kibblewhite, Iain T. McKinnie, James T. Murray, Didier Rabaud, Leslie K. Saddlemyer, Jacques Sebag, James Stillburn, John M. Telle, Jean-Pierre Veran, "MCAO for Gemini South," Proc. SPIE 4839, Adaptive Optical System Technologies II, (7 February 2003); doi: 10.1117/12.459692 Hawai'i, United States 


\title{
MCAO for Gemini-South
}

Brent L. Ellerbroek ${ }^{a}$, François Rigaut ${ }^{a}$, Brian Bauman ${ }^{b}$, Corinne Boyer $^{a}$, Steven Browne ${ }^{c}$, Richard Buchroeder $^{d}$, James Catone ${ }^{a}$, Paul Clark ${ }^{e}$, Céline d'Orgeville ${ }^{a}$, Donald Gavel ${ }^{b}$, Glen Herriot ${ }^{f}$, Mark R. Hunten ${ }^{a}$, Eric James ${ }^{a}$, Edward Kibblewhite ${ }^{g}$, Iain McKinnie ${ }^{h}$, James Murray $^{i}$, Didier Rabaut ${ }^{j}$, Leslie Saddlemyer ${ }^{f}$ Jacques Sebag $^{a}$, James Stillburn ${ }^{f}$, John Telle ${ }^{k}$, and Jean-Pierre Veran ${ }^{f}$

\author{
${ }^{a}$ Gemini Observatory, 670 N. A'ohoku Pl., Hilo, HI 96720 \\ ${ }^{b}$ Lawrence Livermore National Laboratory, ${ }^{c}$ The Optical Sciences Company, ${ }^{d}$ Optical Design \\ Service, ${ }^{e}$ Department of Astronomy, Durham University, ${ }^{f}$ Herzberg Institute of Astrophysics, \\ National Research Council (Canada), ${ }^{g}$ Department of Astronomy, University of Chicago, ${ }^{h}$ Coherent \\ Technologies, Incorporated, ${ }^{i}$ Lite Cycles, ${ }^{j}$ SHAKTIWARE, ${ }^{k}$ Starfire Optical Range, US Air Force \\ Research Laboratory
}

\begin{abstract}
The multi-conjugate adaptive optics (MCAO) system design for the Gemini-South 8-meter telescope will provide neardiffraction-limited, highly uniform atmospheric turbulence compensation at near-infrared wavelengths over a 2 arc minute diameter field-of-view. The design includes three deformable mirrors optically conjugate to ranges of $0,4.5$, and 9.0 kilometers with 349, 468, and 208 actuators, five 10-Watt-class sodium laser guide stars (LGSs) projected from a laser launch telescope located behind the Gemini secondary mirror, five Shack-Hartmann LGS wavefront sensors of order 16 by 16, and three tip/tilt natural guide star (NGS) wavefront sensors to measure tip/tilt and tilt anisoplanatism wavefront errors. The WFS sampling rate is $800 \mathrm{~Hz}$. This paper provides a brief overview of sample science applications and performance estimates for the Gemini South MCAO system, together with a summary of the performance requirements and/or design status of the principal subsystems. These include the adaptive optics module (AOM), the laser system (LS), the beam transfer optics (BTO) and laser launch telescope (LLT), the real time control (RTC) system, and the aircraft safety system (SALSA).
\end{abstract}

Keywords: Multi-conjugate adaptive optics, laser guide stars, adaptive optical systems

\section{INTRODUCTION}

The Gemini Multi-Conjugate Adaptive Optics (MCAO) design is a planned upgrade to the Gemini-South Adaptive Optics (GSAO) system that will provide uniform, diffraction-limited image quality at near IR wavelengths over an extended field-of-view. This will yield a level of scientific utility and observational efficiency well beyond what is feasible with conventional adaptive optics, and provide unique capabilities for Gemini-South in the 2006-9 time frame. For a range of criteria, mean performance over a one square arc minute field-of-view will be comparable to or slightly better than the on-axis performance of a conventional laser guide star (LGS) AO system, such as the LGS upgrade to the Altair AO system on Gemini-North. Highly useful levels of atmospheric turbulence compensation will be achieved over a full two arc minute diameter field-of-view, the maximum possible with the vignetting imposed by the interface to the Gemini instrument support structure (ISS). Sky coverage will be comparable with conventional LGS AO or somewhat superior.

The Gemini-South MCAO design largely eliminates the impact of anisoplanatism on AO performance by compensating atmospheric turbulence in three dimensions. This is accomplished by using three deformable mirrors (DM's) conjugate to distinct ranges in the atmosphere, which are controlled with commands computed from wave front sensor (WFS) measurements of five laser guide stars and three natural guide stars. Table 1 summarizes some of the first order design parameters for these mirrors, sensors, and lasers. These elements have been embodied in a preliminary design of a practical AO system suitable for use with the Gemini-South telescope. The basic approaches and a majority of the components utilized are highly comparable with existing $\mathrm{AO}$ systems, and the system architecture is a natural generalization of current LGS AO approaches. In fact, the proposed design includes a conventional LGS AO system as 
a subset, which will be fabricated and tested at Gemini-South as the first step towards implementing MCAO. There is no provision for conventional natural guide star (NGS) AO or NGS MCAO.

Figure 1 illustrates the principal interactions between the primary subsystems of the MCAO design. These include the laser system (LS), beam transfer optics (BTO), laser launch telescope (LLT), adaptive optics module (AOM), real-time controller (RTC), and aircraft safety and laser traffic control functions (SALSA). The remainder of this paper provides a brief introduction to the requirements, designs, and performance characteristics for these subsystems, preceded by a short summary of system-level performance estimates and some of the science applications for MCAO. Further details may be found in the accompanying paper describing the opto-mechanical design of the $\mathrm{AOM}^{1}$ and in the documents archive at the Gemini Observatory AO web pages. ${ }^{2}$

\section{PERFORMANCE ESTIMATES}

The expected performance of the MCAO system parameters described in Table 1 has been optimized and evaluated through trade studies involving a variety of adaptive optics analysis and simulation $\operatorname{codes}^{3,4,5}$. The predicted RMS wavefront error with median seeing and bright natural guide stars is 252 $\mathrm{nm}$, which corresponds to Strehl ratios of 0.20, 0.40 , and 0.60 in the $\mathrm{J}, \mathrm{H}$, and $\mathrm{K}$ spectral bands. This is an estimate of the average performance over

\begin{tabular}{|c|c|c|}
\hline \multicolumn{3}{|l|}{ Laser Subsystems (Laser, LLT, BTO): } \\
\hline Number of LGS & \multicolumn{2}{|l|}{5} \\
\hline LGS locations in field & \multicolumn{2}{|c|}{$\left(0^{\prime}, 0^{\prime}\right),\left(+/-30^{\prime},+/-30^{\prime}\right)$} \\
\hline LGS signal level at WFS $\left(\mathrm{Na}=2 \mathrm{e} 9 / \mathrm{cm}^{2}\right)$ & \multicolumn{2}{|c|}{390 counts/subap/frame } \\
\hline Transmitted beam quality & \multicolumn{2}{|c|}{1.5 times diffraction limited } \\
\hline RMS 1-axis beam jitter on sky & \multicolumn{2}{|c|}{$0.05 "(0.025 "$ goal $)$} \\
\hline Transmitted beam $1 / \mathrm{e}^{2}$ diameter & \multicolumn{2}{|l|}{$0.3 \mathrm{~m}$} \\
\hline Launch telescope aperture, location & \multicolumn{2}{|c|}{$0.45 \mathrm{~m}$, on-axis } \\
\hline \multicolumn{3}{|l|}{ AO Module: } \\
\hline Number of DM's & \multicolumn{2}{|l|}{3} \\
\hline DM conjugate ranges & \multicolumn{2}{|c|}{$0.0,4.5,9.0 \mathrm{~km}$ (fixed) } \\
\hline Actuator pitch (output space) & \multicolumn{2}{|c|}{$\mathrm{D} / 16, \mathrm{D} / 16, \mathrm{D} / 8$} \\
\hline Number of WFS & $5(\mathrm{LGS})$ & 3 (NGS) \\
\hline WFS order & 16 by 16 & Tip/tilt \\
\hline WFS pixel subtense on sky & $1.0 ”$ & $0.5 ”$ \\
\hline \multirow{4}{*}{$\begin{array}{l}\text { WFS read noise } \\
\text { AOM transmittance to WFS } \\
\text { WFS detector quantum efficiency } \\
\text { WFS sampling rate }\end{array}$} & 6 electrons & None \\
\hline & 0.65 & $0.46-0.69$ \\
\hline & 0.95 & 0.6 \\
\hline & \multicolumn{2}{|l|}{$800 \mathrm{~Hz}$} \\
\hline \multicolumn{3}{|l|}{ Control System (Real time control): } \\
\hline Control algorithm & Zonal (LGS) & 5 modes (NGS) \\
\hline Processing latency + WFS read time & $<1.25 \mathrm{~ms}$ & $<<1.25 \mathrm{~ms}$ \\
\hline$-3 \mathrm{~dB}$ closed loop bandwidth & $32 \mathrm{~Hz}$ & 0-90 Hz, Adjustable \\
\hline
\end{tabular}

Table 1: Selected AO subsystem parameters. $D=7.9 \mathrm{~m}$ is the Gemini aperture.

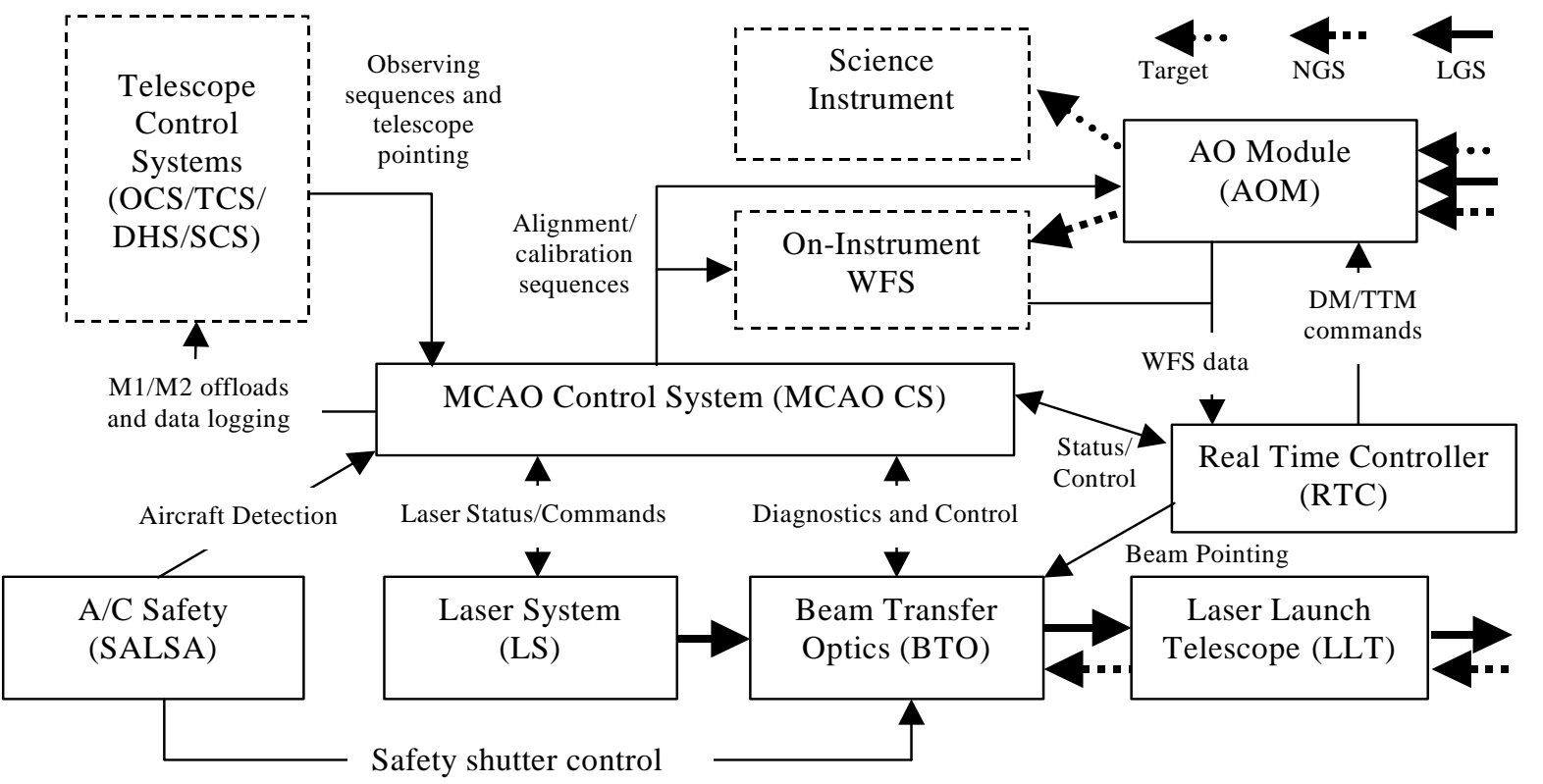

Figure 1: MCAO system block diagram. Note that the real time controller actually offloads tip/tilt commands to the secondary mirror (M2) directly, not through the MCAO CS as illustrated above (to avoid the confusion of intersecting signals). 
the central 1 square arc minute field of view, and includes both basic adaptive optics error sources, higher order effects, and practical limitations of the opto-mechanical and control systems. This section briefly outlines the relative contribution of these various terms.

Figure 2 plots the MCAO Strehl ratio due to combined effect of the fundamental first-order AO error sources as a function of the seeing parameter $\mathrm{r}_{0}$. The median value of $\mathrm{r}_{0}$ at Gemini-South is $0.15 \mathrm{~m}$ for a wavelength of $0.5 \mu \mathrm{m}$, and the $80 \%$ value is about $0.19 \mathrm{~m} .{ }^{6}$ The four first-order error sources, and their contribution to the RMS wavefront error, are: Anisoplanatism over the 1 arc minute square field, $133 \mathrm{~nm}$; fitting error due to the finite spatial resolution of the deformable mirrors and wavefront sensors, 109nm; WFS measurement noise, $32 \mathrm{~nm}$; and the finite temporal bandwidth of the control system (or servo lag), $26 \mathrm{~nm}$. The small values for the last two contributors provide margin for either reduced laser power, increased laser spot size on the sky, or above-average wind velocity-the Strehl ratio reduction due to a $36 \%$ drop in laser power is less than $2 \%$ in $\mathrm{K}$ band and about $4 \%$ in $\mathrm{J}$. The RMS relative Strehl variability over the 1 arc minute square field is about $5 \%$ in $\mathrm{K}$, and $15 \%$ in $\mathrm{J}$, with median seeing.

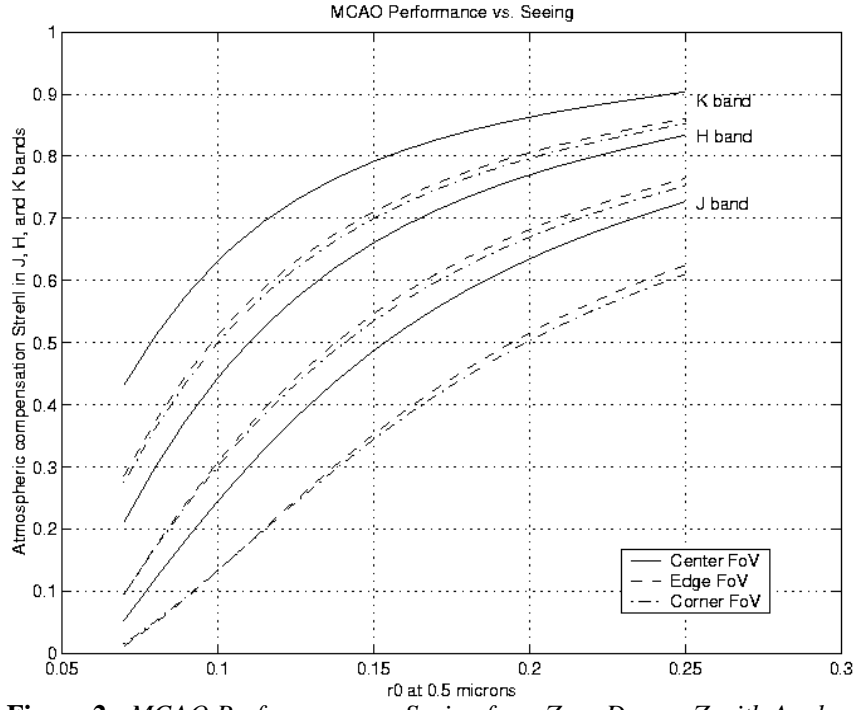

Figure 2: MCAO Performance vs. Seeing for a Zero Degree Zenith Angle and Bright NGS

The remainder of the estimated $252 \mathrm{~nm}$ RMS wavefront error is budgeted towards higher order effects and a variety of implementation error sources. These include physical optics effects associated with diffraction, quadrant detector Shack-Hartmann sensors, and three-dimensional laser guide stars (estimated at $48 \mathrm{~nm}$ RMS via detailed simulation); DM-to-WFS misregistration (estimated at $24 \mathrm{~nm}$ via simulations based on preliminary alignment error budgets); LGS focus variability (10 $\mathrm{nm}$ based on measurements of sodium layer variability); uncalibrated non-common-path errors in the AO Module (46 nm, based on preliminary alignment error budgets); uncorrectable mirror fabrication errors (preliminary budget of $43 \mathrm{~nm}$ ); residual windshake (preliminary estimate of $34 \mathrm{~nm}$ based upon Gemini North wind models), and allocations for uncorrectable errors in the telescope and instrument (116 and $65 \mathrm{~nm}$, respectively). The overall contribution from all of these terms is about $160 \mathrm{~nm}$ RMS, which is modestly smaller than the total error due to the four fundamental error sources as outlined above. By far the largest single contributor is the $116 \mathrm{~nm}$ allocated for uncorrectable telescope errors. Commissioning the Altair AO system at Gemini North later this year will help to characterize and perhaps reduce the magnitude of this term.

Finally, the results plotted in Figure 2 assume that three bright natural guide stars are available within the full MCAO field of view to correct atmospheric tip/tilt, telescope windshake, and tilt anisoplanatism (the requirement for three wellspaced natural guide stars to sense and correct tilt anisoplanatism is one of the major differences between MCAO and conventional LGS AO). Simulations indicate that the limiting NGS magnitude at which the field-averaged Strehl ratio in $\mathrm{H}$ band is reduced by $50 \%$ is about 19.2 to 19.6 , depending upon sky background. Table 2 summarizes the probability of finding three stars of various brightnesses in science field selected at random. Sky coverage is acceptable at the Galactic pole and excellent at 30 degrees galactic latitude, and is superior to our estimates for conventional LGS AO in both cases. ${ }^{7}$ Useful levels of sky coverage are achieved at magnitude 18.5, providing margin for reduced throughput to the NGS WFS.

\begin{tabular}{|l|c|c|c|c|c|c|}
\hline Star Magnitudes & 3 by 18.5 & 3 by 19.0 & 3 by 19.5 & 3 by 20.0 & 17.5 and 2 by 19.5 & 18.5 and 2 by 19.5 \\
\hline $\begin{array}{l}\text { 30 degrees Galactic } \\
\text { Latitude }\end{array}$ & 0.58 & 0.69 & 0.77 & 0.82 & 0.755 & 0.768 \\
\hline Galactic Pole & 0.085 & 0.135 & 0.185 & 0.240 & 0.160 & 0.190 \\
\hline
\end{tabular}

Table 2: Probability of Detecting Three Guide Stars Adequately Placed within a 2.2 Arc Minute Field 


\section{SCIENCE APPLICATIONS}

MCAO is an entirely new tool that offers new research opportunities in astronomy. Its capabilities include the capabilities of classical NGS or LGS AO, but go much further by giving access to large fields of view with relatively uniform image quality. Because they are still immature in their implementation (with the notable exception of the Lick LGS AO system), LGS systems have not yet produced many science results. Although the science produced to date with NGS systems is of quality and very promising, science applications are still limited due to the very stringent sky coverage and field of view limitations, coupled with data reduction difficulties imposed by the variations of the PSF over the field. The latter problem is actually more difficult to solve than it appears, as (a) the problem is usually under constrained because data are lacking to parametrize the PSF variation and (b) to a lesser extent, there was until recently no easy way to implement deconvolution with a field dependant PSF (a new method is proposed by T.Lauer, see "Deconvolution by a spatially variant PSF," this conference). MCAO should, to a large extent, solve all of these problems.

An effort to investigate what kind of science MCAO would enable was started as early as mid-1999. A significant amount of effort went into establishing a MCAO science case-including detailed numerical simulations of such problems as photometry in crowded fields and retrieving shape parameters for distant galaxies-culminating in October 2000 in an workshop held at the headquarter of the Center for Adaptive Optics in Santa Cruz. Approximately 45 astronomers participated. Its goals were "to explore the scientific opportunities for MCAO, quantify its advantages over current and planned conventional AO systems for a comprehensive set of science cases, and derive the MCAO instrument requirements." The discussions were broken into three main groups, addressing the following themes: "The evolution of the mass function of stars in the milky way and the Magellanic clouds", "History and evolution of star formation in nearby galaxies" and "Star formation and history of distant galaxies," coordinated by Pat Roche, Simon Morris, the late Bob Schommer, Taft Armandroff and Tod Lauer, together with others. This process resulted in a comprehensive science case document, available upon request (email to frigaut@gemini.edu), which provides details of over a dozen of science cases, strongly leaning toward star formation research but spread over the entire range of redshift from the milky way to distant galaxies. This opening of sudden interest for AO science at large redshift has clearly been triggered by the new capabilities offered by MCAO.

It is unfortunately impossible to enter into details of the science case here. In essence, the milky way group concentrated on a single, but ambitious, project, which is the determination of the initial mass function of stars in various environments in the Galaxy and the Magellanic clouds. Emphasis was also placed on the importance-and newness-of high precision astrometry on nearby galactic clusters to determine membership and infer their kinematical and dynamical properties. The science in the nearby galaxies theme is primarily derived from the ability to resolve individual stars in galaxies up to the distance of Virgo. The uniform PSF delivered by MCAO will increase the photometric accuracy over a field of view that matches the size of both nearby extragalactic resolved star clusters and the size of galaxies within $100 \mathrm{Mpc}$. Science cases include "calibration of the SNIa zero point," "stellar populations in nearby starburst regions," "the early history of nearby galaxy spheroids," "intergalactic stars," and "extragalactic globular clusters."

The distant galaxy group discussed cases as varied as "field galaxy imaging," "chemical evolution of galaxy disks," "the masses of galaxies at $\mathrm{z}=2$," "galaxy formation in clusters," and "gravitational lensing studies of high redshift galaxies," to address the broad topic of field and cluster galaxy formation and evolution.

Characteristics of the MCAO instruments were derived from the science cases. Two main instruments surfaced: a Near infrared MCAO imager, covering 80"x80" with 20 milliarcsec sampling (currently in the design study phase) and a multi-object spectrograph (the currently planned FLAMINGO-II). A deployable multi-IFU spectrograph was also discussed; however, in the light of its complexity, it was recommended that this instrument be discussed at the meeting for second-generation Gemini instruments, to be held in mid-2003 in Aspen.

\section{ADAPTIVE OPTICS MODULE}

The Adaptive Optics Module (AOM) includes all of the optics, sensors, and electronics needed to compensate the input $\mathrm{f} / 16$ science beam and relay it to a science instrument at $\mathrm{f} / 33$. These components include the principal elements of the real-time Multi-Conjugate Adaptive Optics (MCAO) control loop, namely 3 deformable mirrors, a tip/tilt mirror, 5 
higher-order LGS wave front sensors, 3 tip/tilt NGS wave front sensors, and the real-time control electronics. The AOM is mounted to the Gemini Instrument Support Structure (ISS).

As illustrated in Figure 3, the adaptive optics module has three independently mounted sections. The center bay is for the optics bench, and the two outer bays are for the electronics enclosures. All three bays are mounted, one at a time, onto the Instrument Support Structure (ISS). Some of the basic design characteristics of the optics bench are summarized below. Further details on the complete AOM may be found in an accompanying paper in this volume. ${ }^{1}$

The Optical Bench consists of the opto-mechanical components of the AOM and the associated sensors, mechanisms, and motors. Figure 4 is a schematic illustrating its principal optical components. Beyond those already mentioned, these components include offaxis parabolas to first recollimate and then refocus the input beams, beamsplitters to separate the science, NGS, and LGS optical paths, atmospheric dispersion correctors (ADC's) in the science and the NGS WFS paths, and two subsystems for WFS and DM calibration:

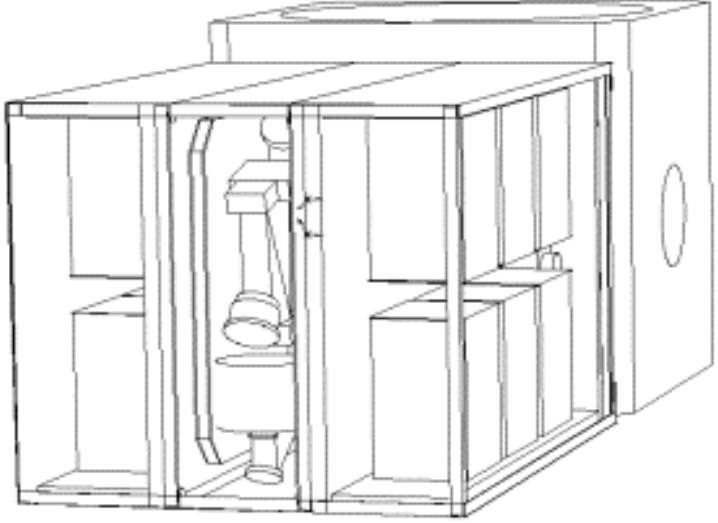

Figure 3: AO Module mounted on the ISS, showing the optics bay in the center and flanked by the two electronics enclosures. Some outer panels have been omitted for this illustration

1. Simulated natural and laser guide stars located at the Cassegrain focus near the entrance of the AOM. These simulated guide stars are used for (i) verification of optical alignment between AOM and science instruments, (ii) PSF and field distortion calibrations, (iii) measurement of DM influence functions and DM-to-WFS alignment, and (iv) closed-loop tests of the MCAO control loop.

2. A higher-order WFS located in the output science path. This sensor patrols the 1 arc minute radius field, and is used for diagnostic wave front measurements with the NGS source simulator. These measurements provide an end-to-end evaluation of AO module ontical performance. and thev are used as invut to a "tomographic" wave front reconstruction algorithm errors in the science path. This sensor in effect substitutes for the "DM interferometer" found in several existing AO systems. Whether this diagnostic sensor will be implemented in the field, or only used for initial system characterization in the lab, is still to be determined.

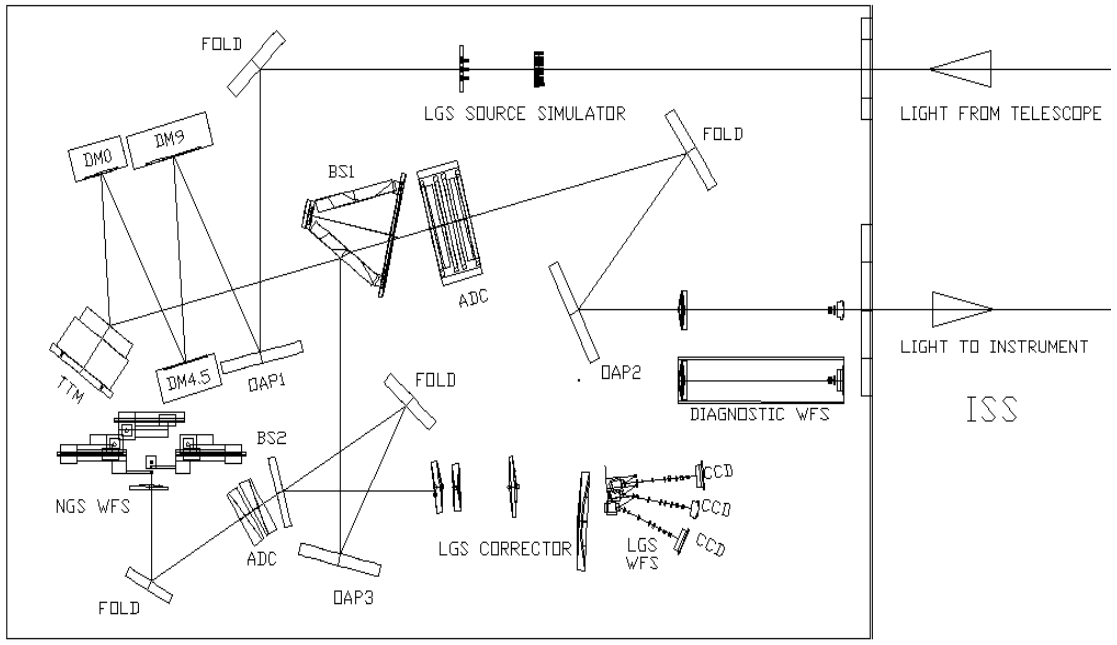

Figure 4: AO Module optical schematic 


\section{REAL-TIME CONTROL SYSTEM}

The Real-Time Control System is the heart of the Adaptive Optics System, and the most critical in terms of real time performance. It will handle 3 basic real time functions:

- The LGS real time control process. This process reads the 5 LGS wavefront sensors, computes the slope measurements, and computes the commands for the 3 deformable mirrors. This process is executed at a rate of $800 \mathrm{~Hz}$, and is therefore the most critical in terms of real time performance. The number of operations required for this process is around 2.26Gflops, or 1.13 Giga multiply/accumulate (MAC) operations per second.

- The NGS real time control process. This process reads the 3 NGS wavefront sensors, computes the tip-tilt measurements, and computes the tip-tilt and DM mirror commands to compensate global tip-tilt and tilt anisoplanatism. This process is executed at a rate of $800 \mathrm{~Hz}$, and is not a critical process in terms of real time performance. The total number of operations required for this process is around 3.16Mflops, or 1.58M MAC per second

- The optimization and background processes. The purpose of these processes is to continuously optimize or update the parameters of the NGS and LGS control processes (e.g., the temporal filter parameters), and also provide control for external components such as the BTO Fast Steering Array. These processes are also closed loop processes, but run at a slower rate than the LGS and NGS processes.

The requirements for the Real-Time Control System have been described in detail previously. ${ }^{8}$ The following paragraphs describe the advances made during the last year in specifying the system architecture.

The Optical Science Company (tOSC), located in Anaheim, California, has been contracted to build the Real Time Controller. The architecture of the Real Time Controller is based on 4 dual PPC boards mounted on a PCI backplane controlled by a separate standard $1.8 \mathrm{GHz}$ Pentium Computer. A description of the architecture proposed by tOSC is given in Figure 5.

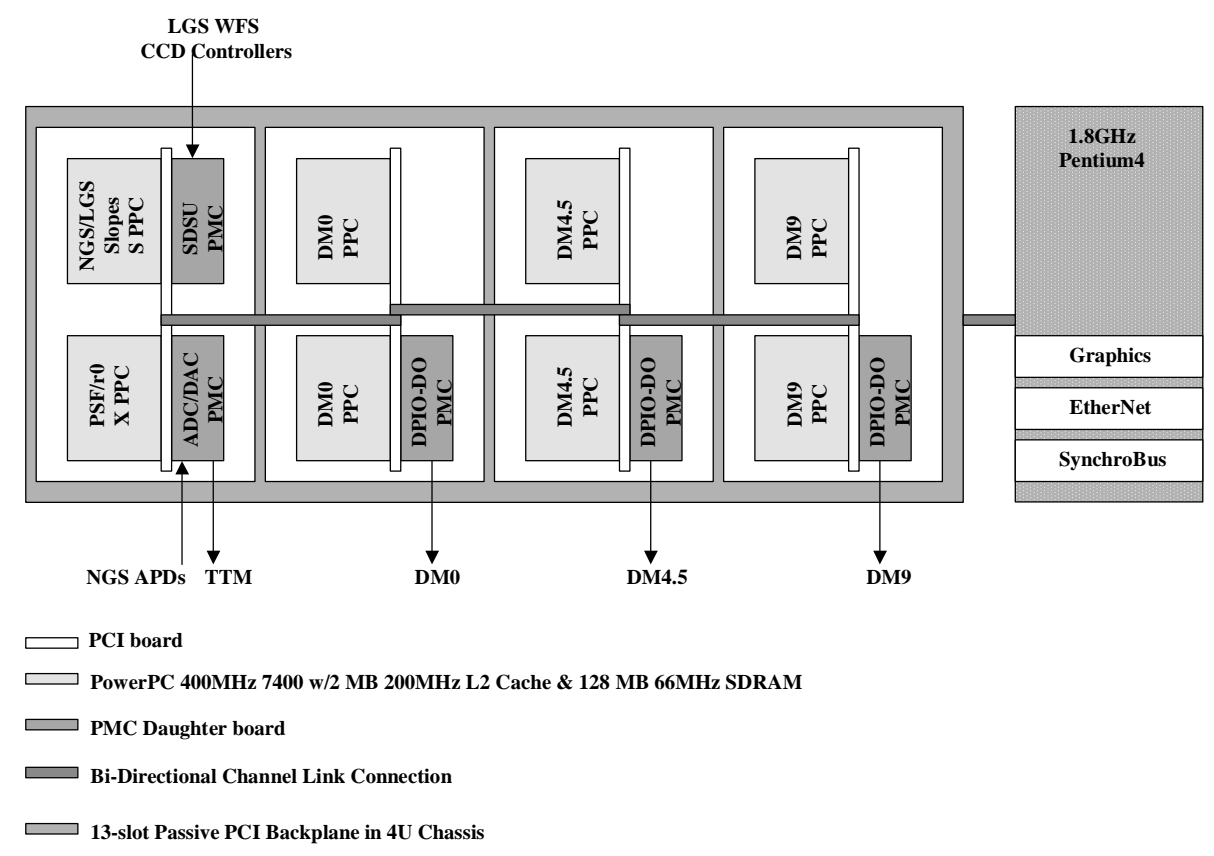

Figure 5: Real Time Controller architecture
The PowerPC boards are Alacron's Fast Vision boards. Each board contains two PowerPCs 7400 processors. Each PowerPC has a dedicated 2MB Level 2 data cache, a dedicated 128MB of SDRAM, a dedicated PMC site, a dedicated 200MB/s FastChannel interface and a dedicated 100MB/s ChannelLink interface. PCI bridges between the boards themselves and with the host computer allow accessing the resources of all the processors and of the host.

tOSC will implement the LGS and NGS controls processes using a parallel architecture to minimize latency in the control loop.

The leftmost PowerPC board is dedicated to the NGS real time process, and to computing LGS slopes as pixels are digitized from the LGS WFS cameras. The resulting LGS slopes and the modal DM adjustments needed to compensate tilt anisoplanatism are passed to the three remaining reconstructor PowerPC boards, one dedicated to each deformable mirror. These three reconstructor boards compute the deformable mirrors commands using fixed-point arithmetic. The 
architecture achieves better than $100 \mu$ s total processing latency for the LGS processing loop, defined as the time between receiving the last WFS pixel value and sending the final actuator command to the deformable mirrors.

All real-time I/O is implemented via the PMC cards piggybacked on the PowerPC boards as illustrated in Figure 5. Most of the optimization and backgrounds tasks are also performed on these four dual PowerPC boards. The maximum load for any of the PowerPC processors is estimated at less than $70 \%$ of the specified capability.

The host Pentium runs Windows 2000 to provide the engineering GUI and the real time graphics displays, and will also host the RTC EPICS software to interface with the remainder of the MCAO Control System.

\section{LASERS}

The MCAO Laser System includes all components, both hardware and software, necessary to produce and maintain five laser beams at the peak wavelength of the sodium D2 line $(589.0 \mathrm{~nm})$. These components are one or multiple laser heads and laser enclosures, the laser electronics, a control system, cooling systems, and any necessary diagnostics. This section briefly reviews the laser system requirements as presently understood, as well as recent progress in sodium laser research and development $(\mathrm{R} \& \mathrm{D})$.

The single most important performance requirement for the Gemini South laser system is obviously the laser output power needed to produce five laser guide stars for MCAO. Depending on mesospheric sodium column density $\left(2-310^{9}\right.$ atoms $\left./ \mathrm{cm}^{2}\right)$, zenith angle $\left(0-45^{\circ}\right)$, and other assumptions, the total laser power requirement will be in the 50W range for continuous-wave $(\mathrm{CW}), \mathrm{CW}$-modelocked, and $800 \mathrm{~Hz}$-macro-micro pulsed laser formats. ${ }^{11,12}$ The use of pulsed lasers with an $800 \mathrm{~Hz}$ repetition rate that enables time-gating of Rayleigh scattered light on the LGS WFS would certainly increase the MCAO system's overall performance and enable operation with subvisible cirrus present, but CW and CWmode-locked lasers have not been ruled out at this time. Other temporal formats that are less efficient in exciting mesospheric sodium atoms will most likely require too high an output power to be seriously considered for the MCAO laser. The final laser power requirement will be determined in time for the MCAO Critical Design Review, based on the results of the Gemini sodium monitoring campaign in Chile ${ }^{13}$ and the desired scientific use of the MCAO capability at Cerro Pachón.

Other laser performance, functional, and operational requirements also include: Output beam formatting for five 1.2 times diffraction-limited beams arranged in an X-shape for interface with the Beam Transfer Optics and delivery at the telescope center section; a user-friendly, fully automated laser system interfaced to the MCAO Control System with all appropriate on-line diagnostics; easy maintenance by a dedicated laser technician and high reliability throughout the night. ${ }^{12}$

A year ago, reference 12 gave an overview of the on-going Gemini laser Research and Development (R\&D) program, and reported on three risk-reduction activities initiated in collaboration with the US Air Force and the Center for Adaptive Optics (CfAO). The diode-pumped solid-state laser R\&D programs initiated by Gemini and others are now bearing fruits. The $\mathrm{CW}$-mode-locked 1.06+1.32 micron sum-frequency risk-reduction program funded by Gemini last year at Coherent Technologies, Inc. (CTI) of Lafayette, $\mathrm{CO}$ has successfully paved the way for a full-scale laser design and fabrication contract for the Gemini North Adaptive Optics system, Altair. The contract, which is currently awaiting final AURA/NSF approval, foresees delivery of an engineered 10-20W class CW-mode-locked laser in early 2004 for acceptance testing followed by commissioning with Altair. Another program, led by the University of Chicago (UoC) in an industrial partnership with Lite Cycles of Tucson, AZ, consisted of refurbishing the UoC $800 \mathrm{~Hz}$ macro-micro pulsed sum-frequency laser to produce higher laser outputs in the 20-40W range. Although delays in the fabrication of three newly-designed gain modules for the 1.06 and 1.32 micron lasers postponed completion of the program, development of the modules is now complete and preliminary tests have demonstrated improved efficiency. The refurbished UoC laser is expected to equip the 200-inch Palomar telescope for use with its Adaptive Optics system in 2003. Finally, the Cooperation and Development Agreement (CraDA) between Gemini and the US Air Force to develop a CW sumfrequency laser was recently renewed. The laser team at Starfire Optical Range (SOR) is obtaining highly encouraging results towards building $10 \mathrm{~W}$ and $50 \mathrm{~W}$ versions of their resonant-ring design. Both versions will be tested on the sky this fall. 
Parallel laser R\&D programs lead by other groups, such as the Lawrence Livermore National Laboratory (LLNL)/European Southern Observatory (ESO) 0.94+1.58 micron sum-frequency fiber laser effort, have also shown very promising results towards building a $10 \mathrm{~W}$ prototype. Even though progress always seems slow when compared to the urgent needs of the community in general and the Gemini Observatory in particular, research in sodium laser R\&D is progressing well, and hope to see powerful, efficient, and reliable sodium lasers in the mid-term future is rising.

\section{BEAM TRANSFER OPTICS AND LAUNCH TELESCOPE}

The Beam Transfer Optics (BTO) is the MCAO subsystem that brings the five laser beams from the Laser System, located on the telescope center section, to the Laser Launch Telescope (LLT) mounted behind the telescope secondary mirror. The Laser Launch Telescope is basically a beam expander which projects the five laser beams arranged in an Xshaped constellation with one on-axis beam and four off-axis beams separated by 42.5 arcsec from the on-axis beam on the sky.

Beam Transfer Optics: The BTO consists of the enclosed optical train from the output of the laser source to the entrance pupil of the LLT. Briefly, the BTO can be thought of in two sections: the first section delivers the 5 beams from the laser source to the BTO Optical Bench (BTOOB) above the telescope secondary without letting scattered light contaminate the LGS WFS's (Figure 6); the second section formats the beams into the 5-beam constellation and aligns the beams into the LLT (Figure 7). Separate control systems guide the control surfaces of each BTO section.

Starting from the laser source, the key BTO components are:

- Two actively-controlled mirror arrays to point and center the beam across the secondary vane

- A relay telescope to relay the pupil of the laser source to the entrance pupil of the LLT; this relay is located on the side of the main telescope

- A fast steering array for active pointing control of each of the laser guide stars on the sky

- An array of static mirrors that reformats the incoming beams into the 5-beam x-shaped constellation seen on the sky

- A diagnostic package that provides feedback for the alignment of the beam over the secondary vane and provides boresighting information between the main telescope and the LLT

- Two actively-controlled mirrors that point and center into the LLT, thus compensating flexure or other commonmode pointing errors

- An actively-controlled, rotating K-mirror that keeps the orientation of the LGS constellation on the sky constant with respect to the WFS.

- Three auxiliary cameras that aid in the coarse alignment of the BTO.

Laser Launch Telescope: The 5 laser beams must be launched on-axis to minimize the perspective elongation of the LGS images. The LLT optical design is therefore constrained by two considerations: (1) the LLT structure must be hidden from the Cerro Pachon telescope field of view and fit inside the telescope secondary frame, and (2) the LLT clear aperture must be as large as possible (on the order of 50 $\mathrm{cm}$ ) in order to create the smallest LGS spot sizes when seeing is good. Additionally, if the laser beams are to be hidden behind one of the secondary

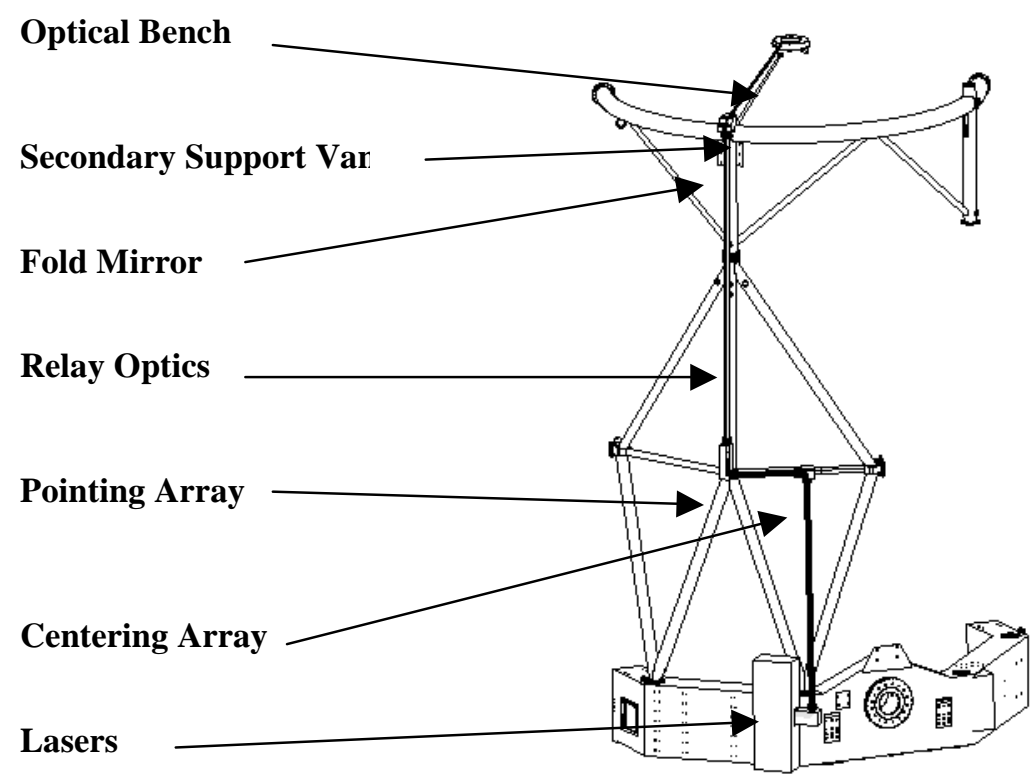

Figure 6: Beam Transfer Optics path from the laser system to the optical bench located behind the Gemini secondary mirror 
vanes, then the beams must be fed into the LLT from the top of the structure, with a maximum beam full diameter (99\% encircled energy criteria) smaller than $10 \mathrm{~mm}$.

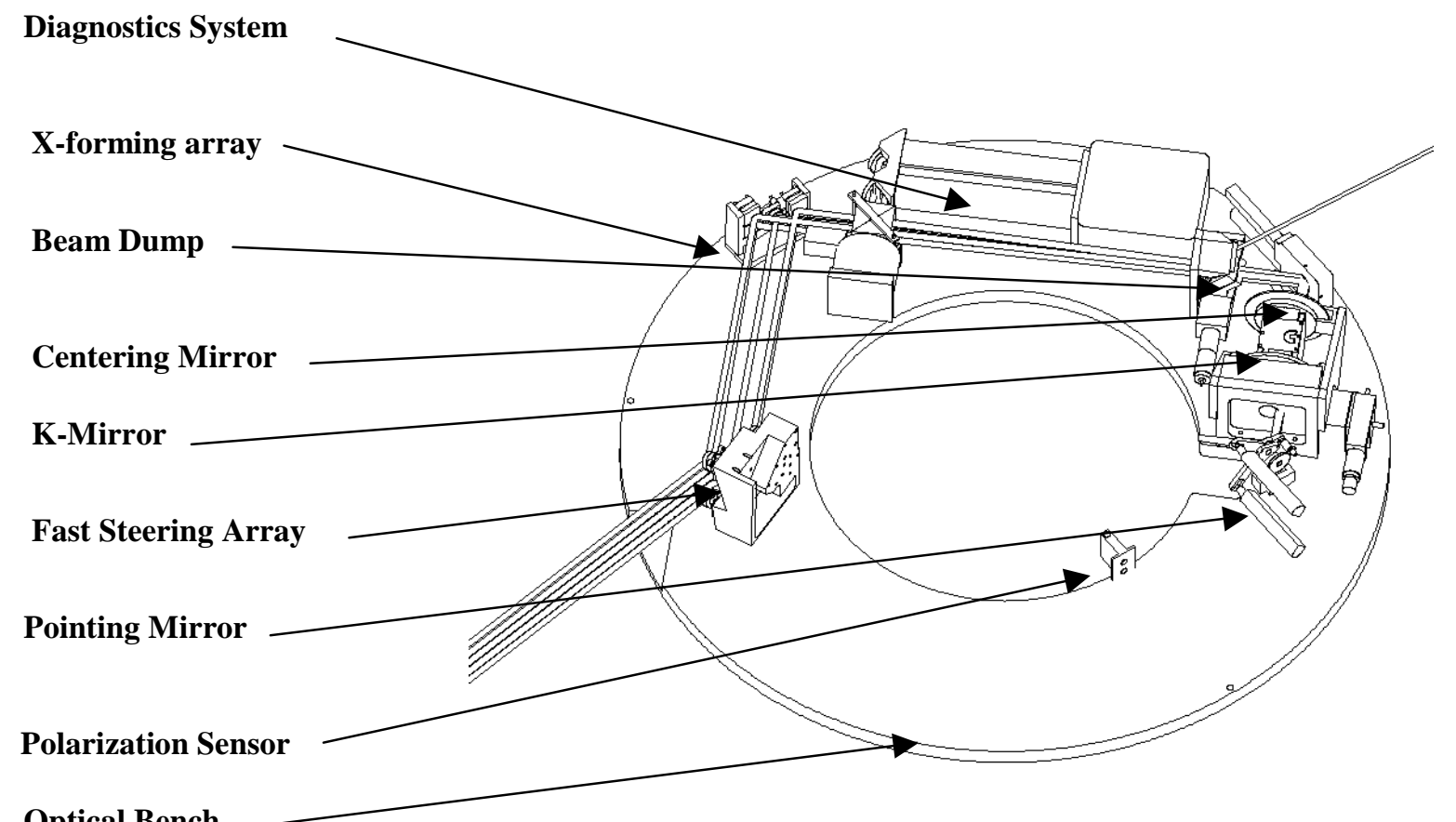

Optical Bench

Figure 7: Beam Transfer Optics Optical Bench

The LLT is currently being designed and built by EOS Technologies of Tucson, Arizona.

LLT Optical Design: The LLT is a true afocal telescope with a $60: 1$ magnification ratio that projects a $450 \mathrm{~mm}$ collimated output beam to the sky. The real input beams will be gaussian beams with a diameter of $5 \mathrm{~mm}$ at the $1 / \mathrm{e}^{2}$ intensity points corresponding to a projected $300 \mathrm{~mm}$ diameter at the $1 / \mathrm{e}^{2}$ intensity points. The telescope consists of an aspheric negative lens doublet, a folding flat and an off-axis parabolic (OAP) primary mirror. The total laser power is expected to be around $50 \mathrm{~W}$, with considerably higher peak power if the MCAO laser system is pulsed. Because of high laser power density at the small lenses in the $500-1000 \mathrm{~W} / \mathrm{cm}^{2}$ range, attention is being given to resistance of the glass itself, and to adhesion of the antireflection coatings.

LLT Mechanical Design: The LLT, as designed by EOS Technologies and illustrated in Figure 8, is basically a cylinder with the OAP primary mirror at one end and a flange on the other end. The flange attaches the telescope to the top of the Gemini telescope secondary support structure. There will be an additional lateral support system at the lower end. The telescope is made primarily of aluminum with a carbon fiber thermal compensation rod to maintain the primary mirror to beam expander distance.

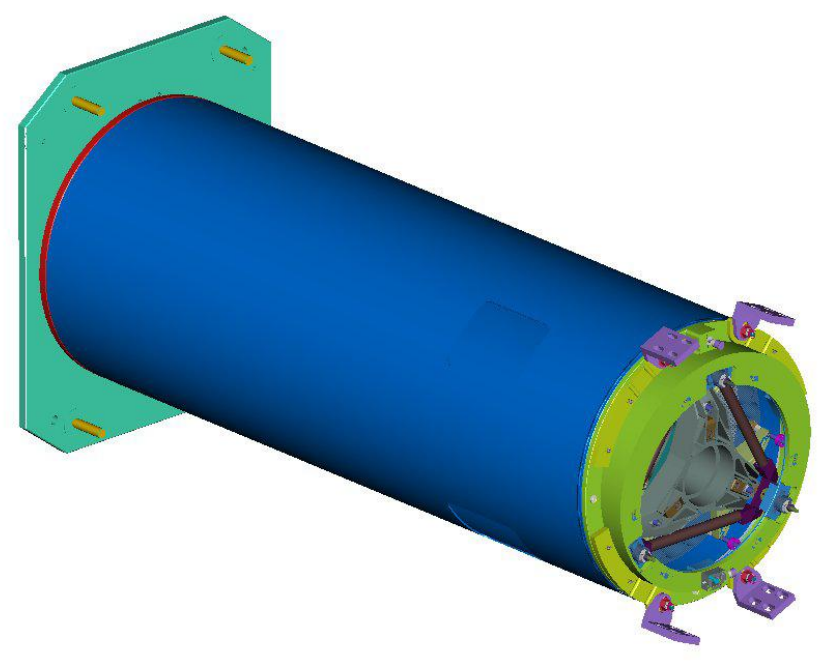

Figure 8: LLT Mechanical Layout 


\section{AIRCRAFT SAFETY AND LASER TRAFFIC CONTROL}

The laser safety systems for Gemini laser guide star program are grouped under the SALSA acronym for Safe Aircraft Localization and Satellite Avoidance. The primary components of the SALSA system are:

- Human and equipment safety features implemented as part of the laser system design and operating procedures. These include enclosures along the entire beam path, automatic safety shutters and interlocks, the use of specific procedures and eyewear during maintenance, and the choice of a solid-state laser design to eliminate chemical and fire hazards.

- A reliable aircraft detection system. This includes an all-sky camera at the summit to warn the observer of approaching aircraft, and a boresighted camera mounted on the telescope to automatically control a safety shutter when an aircraft approaches the beam.

- A communications interface with the Laser Clearinghouse at US Space Command. Like Lick and Keck Observatories, Gemini will comply with the requirements and procedures originally developed to avoid accidental illumination of domestic and foreign satellites by US Department of Defense laser systems. We also intend to open discussions with the Laser Clearinghouse to clarify their charter with respect to civilian laser propagations, investigate if the frequency of propagation blackouts can be reduced, and to upgrade communication interfaces.

- A Laser Traffic Control System (LTCS) to avoid beam collisions with other observatories. Keck and Gemini Observatories have implemented and successfully tested the initial version of this system for the Mauna Kea summit, and will continue working together to improve the system's efficiency and reliability. ${ }^{9}$

Human and equipment safety: Control measures are designed to reduce the possibility of exposure of the eye and skin to hazardous levels of radiation, and to limit non-beam hazards during operation and maintenance. The laser and the beam path will be enclosed to minimize the exposure hazard. A special enclosure will also be mounted on the laser during maintenance to isolate it from the remainder of the dome. Specific operating procedures will be followed before, during, and after laser propagation, and personnel will wear protective glasses. Gemini will use solid-state laser technology and thus avoid compressed gazes, dyes, or solvent, which will limit chemical hazards. Safety shutters and interlocks are also included in the Laser Guide Star system design (see figure 9). The existing Gemini Interlock System (GIS) is used to control the safety shutter. A hazard evaluation is currently underway to identify all the possible hazardous situations.

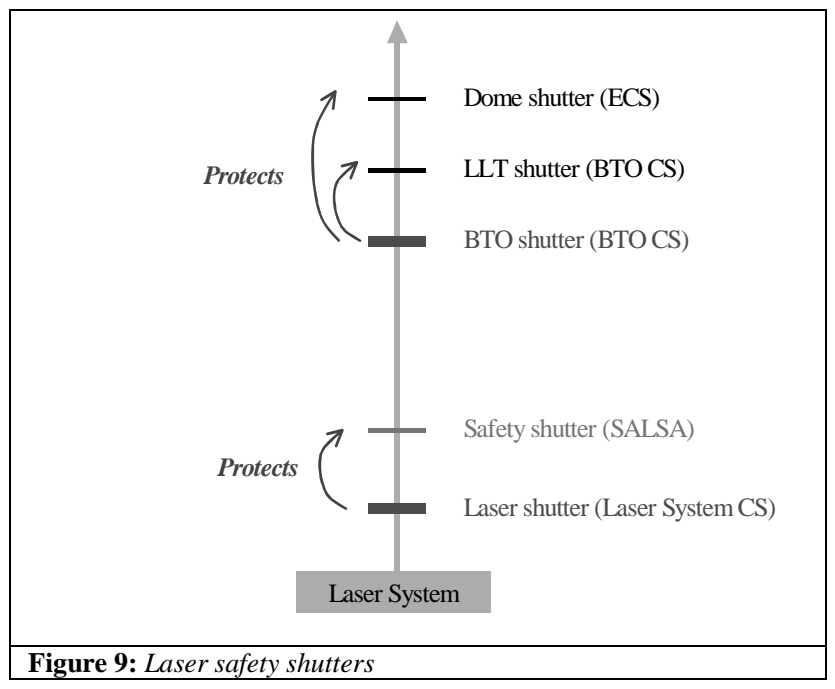

Aircraft detection: The aircraft safety design employs two main levels of detection. The first will provide a warning in case of global aircraft detection. This tool will help in planning observations and for predictive beam avoidance. An allsky camera system located at the summit takes continuous images over a 180 degrees field of view. The images are analyzed in real-time. Software computes the position, apparent velocity, and approximate altitude of the aircraft detected. A prototype system is currently being constructed to validate the design on the sky.

Users will also have an all-sky video display fed with data provided by airport radars. Such a system has already been deployed on Maui. Keck Observatory is leading the effort to enable access by Mauna Kea telescopes to this design work. ${ }^{9}$

A boresighted detection level, within a small field of view centered on the laser beam, will activate the safety shutter to stop laser propagation automatically in case of aircraft detection. The boresighted system is composed of an IR camera mounted on the telescope. The camera has a 20 degree field-of-view centered on the optical axis of the laser launch telescope. The collected imagery is analyzed in real-time. If an aircraft is detected, a signal is sent to close the safety 
shutter located near the output of the laser. The specified time limit to close the shutter is 0.25 seconds. In comparison, the worst-case time for a low-flying aircraft to transit half the field-of-view of the camera is about 1 second.

Satellite predictive avoidance: The Laser Clearinghouse at US Space Command reviews requests for ground-to-space laser propagations from DoD and DoE agencies, including past and ongoing laser guide star research at Lick Observatory in California by Lawrence Livermore National Laboratory. Requests for propagation must be submitted at least 72 hours in advance by fax, and must specify the time, place, and direction of propagation and a brief list of firstorder laser parameters (wavelength, power, divergence, and pulse format). The Laser Clearinghouse compares the request against their database of satellite orbital parameters, and responds (again by fax) with a list of blacked-out times for each proposed laser propagation.

Just as the Lick and Keck Observatories have done, Gemini Observatory intends to submit requests for laser propagations to the US Space Command Laser Clearinghouse and abide by any predictive avoidance blackouts that are indicated. Because the current procedures are labor-intensive and will reduce observing efficiency to a significant extent, Gemini also intends to open communications with the Laser Clearinghouse to clarify the current requirements and explore any possibilities for improvements. The principle issues to be resolved are to clarify the charter and responsibilities of the Laser Clearinghouse with respect to civilian laser systems at US, international, and foreign observatories. Although the Clearinghouse has recently accepted and processed propagation requests from Keck Observatory, there is evidently no explicit public description of the scope of their authority for such systems. We also plan to investigate if the blackout periods can be reduced by diminishing the 15-degree keep-out zone around each satellite of interest. More generally, it may be possible to reduce the number and duration of blackout periods by more carefully modeling the risks associated with 10-Watt-class sodium laser beams on a satellite-by-satellite basis.

Finally, the communication interfaces for laser propagation requests could be modernized. An upgrade from fax to email would represent a significant savings in time for both parties if network security issues can be resolved. A much more ambitious proposal is to allow the Laser Clearinghouse to monitor telescope pointing information continuously and approve laser propagation in real time, using a web-based interface similar to the beam collision avoidance system described below.

Laser traffic control: The need for a beam collision avoidance system was identified early in the effort to permit the use of laser guide stars above Mauna $\mathrm{Kea}^{10}$. Its purpose is to protect all telescopes from beam interference that would impact their science observations. A "collision" occurs when the projected laser beam enters the field of view of an instrument mounted on a second telescope, due to either Rayleigh- or resonant sodium backscatter. A Laser Traffic Control System (LTCS) has been developed at Keck Observatory ${ }^{9}$ with support from Gemini, and has now been successfully tested during the recent Keck laser propagations. The web-based system analyses data provided by a URL at each telescope on the Mauna Kea summit, computes all possible collisions, and signals a laser safety shutter in case of interference.

\section{SUMMARY}

Further detail on all of the above subjects may be found at the Gemini AO web pages. ${ }^{1}$ The overall GSAO project is now transitioning into the detailed design and fabrication phase. The RTC contract has been awarded to the Optical Sciences Company as stated previously, and the detailed design work for the LLT is already well underway. The contract for the AO module optical bench and the NGS wavefront sensors is nearly ready for approval, and design and development efforts for the LGS WFS and the deformable mirrors will be initiated soon. In-house work on the BTO, control system, and safety system is continuing. No new technical risks or unexpected complexities have been uncovered as the design progresses. The availability of a reliable and affordable 50-Watt-class laser system remains the dominant risk item, and $\mathrm{R} \& \mathrm{D}$ work over the past year continues to raise confidence that this requirement will be met.

\section{ACKNOWLEDGEMENTS}

This work was supported by the Gemini Observatory, which is operated by the Association of Universities for Research in Astronomy, Inc., under a cooperative agreement with the NSF on behalf of the Gemini Partnership: the National Science Foundation (United States), the Particle Physics and Astronomy Research Council (United Kingdom), the 
National Research Council (Canada), CONICYT (Chile), the Australian Research Council (Australia), CNPq (Brazil), and CONICET (Argentina).

\section{REFERENCES}

1. E. James, C. Boyer, R. A. Buchroeder, B. L. Ellerbroek, and M. R. Hunten, "Design considerations for the AO Module for the Gemini South multiconjugate adaptive optics system," in Adaptive Optical System Technologies II, SPIE Proc., Vol. 4839, paper 4839-08 (2002).

2. Gemini adaptive optics web pages, http://www.gemini.edu/sciops/instruments/adaptiveOptics/AOIndex.html.

3. B. L. Ellerbroek, "First order performance evaluation of adaptive-optics systems for atmospheric turbulence compensation in extended field-of-view astronomical telescopes," J. Opt. Soc. Am. A 11, 783-805 (1994).

4. R. Flicker, F. J. Rigaut, and B. L. Ellerbroek, "Comparison of multiconjugate adaptive optics configurations and control algorithms for the Gemini South 8-m telescope," in Adaptive Optical Systems Technology, SPIE Proc. Vol. 4007, 1032-1043 (2000).

5. B. L. Ellerbroek, "Wave optics propagation code for multiconjugate adaptive optics," in Adaptive Optics Systems and Technology II, Proc. SPIE 4494, 104-121 (2001).

6. J. Vernin, A. Agabi, R. Avila, M. Azouit, R. Conan, F. Martin, E. Masciadri, L. Sanchez, and A. Ziad, "1998 Gemini site testing campaign: Cerro Pachon and Cerro Tololo," Gemini Doc. RTP-AO-G0094 (Gemini Observatory, Hilo, Hawaii, 2000).

7. F. Rigaut and E. Gendron, "Laser guide star in adaptive optics: the tilt determination problem," Astron. Astrophys. 261, 677-684 (1992).

8. C. Boyer, J. Sebag, M. Hunten, and L. Saddlemyer, "Gemini MCAO Control System, in Adaptive Optics Systems and Technology II, Proc. SPIE 4494, 167-180 (2001).

9. D. Summers, B. Gregory, P. J. Stomski, Jr., A. Brighton, R. J. Wainscoat, P. L. Wizinowich, W. Gaessler, J. Sebag, C. Boyer, T. Vermeulen, A J. Denault, D. A. Simons, H. Takami, and C. Veillet, "Implementation of a laser traffic control system supporting laser guide star adaptive optics on Mauna Kea," in Adaptive Optical System Technologies II, SPIE Proc., Vol. 4839, paper 4839-57 (2002).

10. P. Wizinowich, D. Simons, H. Takami, C. Veillet, and R. Wainscoat, "Coordination and Use of Laser Beacons for Adaptive Optics on Mauna Kea," in Adaptive Optical System Technologies, SPIE Proc., Vol 3353, pp. 290-299 (1998).

11. C. d'Orgeville, F. Rigaut and B. L. Ellerbroek, "LGS AO photon return and laser requirements for the Gemini LGS AO program", in Adaptive Optics Systems and Technology, SPIE Proc., Vol. 4007, pp. 131-141 (2000)

12. C. d'Orgeville, B. Bauman, J. Catone, B. Ellerbroek, D. Gavel, R. Buchroeder, "Gemini North and South Laser Guide Star Systems Requirements and Preliminary Designs", in Adaptive Optics Systems and Technology II, SPIE Proc., Vol. 4494, pp. 302-316 (2001)

13. C. d'Orgeville, M. Boccas, C. Dainty, R. Flicker, B. Gregory, L. Michaille, J. Quartel, F. Rigaut, A. Tokovinin, G. Trancho, N. Wooder, "Preliminary results of the 2001-2002 Gemini sodium monitoring campaign at Cerro Tololo, Chile", in these proceedings (2002) 